\title{
A NATURAL POPULATION OF DROSOPHILA TRANSFERRED TO A LABORATORY ENVIRONMENT
}

\author{
W. W. ANDERSON, TH. DOBZHANSKY and O. PAVLOVSKY \\ Department of Biology, Yale University, New Haven, Connecticut 06520; \\ and Department of Genetics, University of California, Davis, California 95616
}

Received 2.iv.71

\section{INTRODUGTION}

ExPERIMENTs have been made with several species of Drosophila, testing the fitness of genetic variants found in free-living populations. In particular, gene arrangements in the third chromosomes of Drosophila pseudoobscura have been made to compete in laboratory population cages, kept at various temperatures and fed on culture media inoculated with different species of yeasts (Pavlovsky and Dobzhansky, 1966; Dobzhansky, 1970; and references therein). However, with the exception of the study of Anderson, Dobzhansky and Kastritsis (1967), the relative frequencies of the chromosomal variants, or of the species, among the founders of the experimental populations were different from those in the ancestral natural populations. The founders also included less than the complete range of the chromosomal variants, or of the species, present in nature. These technical details may not be trivial. In several experiments (Levene, Pavlovsky and Dobzhansky, 1958; Anderson et al., 1968; Watanabe et al., 1970) evidence was obtained that the Darwinian fitness of the karyotypes may be dependent on which genetic variants are represented in a given population, and may also be frequency-dependent.

In the present article we report the results of experiments in which the founders of the experimental laboratory populations were deliberately chosen to reflect the composition of the ancestral natural populations.

\section{Materials and technique}

Samples of the Drosophila population were taken between 29th June and 5th July 1969, at Mather, Tuolumne County, California, by Dr F. J. Ayala, Mrs M. H. Ayala, and Th. Dobzhansky. The flies were attracted to fermenting banana bait in late afternoons and up to half an hour after sunset. A partial count showed the following frequencies of species:
D. pseudoobscura, D. persimilis, D. azteca 우우 513
D. pseudoobscura, D. persimilis ơ 010
D. azteca $\widehat{\delta} \widehat{0}$
D. miranda 우 and $\bar{\delta} \sigma^{\circ}$
D. pinicola 우 and $\hat{\sigma} \hat{\sigma}^{-1}$
D. occidentalis 우 and $\widehat{\partial} \hat{\sigma}$
Other species

Males of $D$. pseudoobscura and $D$. persimilis are not easily distinguishable, and are scored jointly. Females of these species are not distinguishable by quick inspection from $D$. azteca, and are also scored together. $D$. pinicola and $D$. occidentalis are fungus-eaters, and probably are not ecological competitors of the species of the obscura group. These latter were brought to the 
laboratory in New York, and the females were placed singly in culture bottles and allowed to produce offspring. A mature larva from each bottle was dissected and its salivary glands stained in acetic orceine. The cytological diagnosis of the species is easy and reliable.

When the adult flies emerged in the cultures, they were etherised, and about equal numbers of females and males were taken from each culture to serve as founders of the populations in the experimental population cages. Two replicate populations (I and II) were set up. Plastic population cages $\left(15^{\prime \prime} \times 11^{\prime \prime} \times 5 \frac{1}{2}\right.$ ", Takashima Co., Japan) with 15 containers of culture medium were used. A new container with fresh medium was inserted three times per week. The populations were kept in a constant temperature room at $25^{\circ}$ C. Spassky's cream of wheat-molasses medium, inoculated with Fleishman's yeast, was used throughout.

\section{EXPERIMENTAL RESULTS}

Among the 500 progenies of the flies collected in Mather, the following species were found when tested cytologically:

$\begin{array}{lr}\text { D. pseudoobscura } & 156 \\ \text { D. persimilis } & 283 \\ \text { D. miranda } & 28 \\ \text { D. azteca } & 33\end{array}$

The two experimental populations were set up with the following numbers of founders of each species:

$\begin{array}{lcccc} & \text { D. persimilis } & \text { D. pseudoobscura } & \text { D. miranda } & \text { D. azteca } \\ \text { Population I } & 2000 & 1000 & 250 & 80 \\ \text { Population II } & 2000 & 1000 & 250 & 120\end{array}$

The small numbers of $D$. azteca among the founders were due to a low fertility of the females of this species in laboratory cultures. No D. azteca or $D$. miranda adults hatched in the population cages. D. persimilis is a weak competitor with $D$. pseudoobscura in the experimental environment. Samples of eggs were taken in the populations 35 and about 90 days after the start, and the chromosomes in the salivary glands of mature larvae were studied. Population I had 150 D. pseudoobscura and no D. persimilis at 90 days. Population II was slightly different; at 35 days, there were $150 \mathrm{D}$. pseudoobscura, $7 \mathrm{D}$. persimilis, and 1 hybrid larva; at 96 days there were $150 \mathrm{D}$. pseudoobscura, I $D$. persimilis, and 1 hybrid. All the samples after 90 days contained only D. pseudoobscura larvae.

The appearance in population II of hybrids between $D$. pseudoobscura and $D$. persimilis is of interest. The ethological isolation between these species is far from complete in laboratory environments, although effectively so in nature (Dobzhansky, 1951, and later unpublished observations). In the population cages, both $D$. persimilis and the hybrids are rapidly eliminated; none appeared in the sample at 155 days or later. Introgressive hybridisation of species of Drosophila has been observed in the carefully conducted experiments of Mettler (1957). He had population cages with females and males of $D$. mojavensis and $D$. arizonensis as founders; a hybrid population eventually supplanted the pure species. It should be noted that $D$. mojavensis and $D$. arizonensis are allopatric in distribution, so that their hybridisation 
in nature is excluded by geographic separation. By contrast, $D$. pseudoobscura and $D$. persimilis are often sympatric but not interbreeding in nature. Van Valen (1963) reported that a "hybrid swarm " was formed in population cages with a mixture of the two species held at $25^{\circ} \mathrm{C}$. Our data provide no support for this claim, but they are not necessarily in contradiction. Among other differences in experimental designs, our experiments used progenies of wild-collected flies, while those of Van Valen used mutants which had been kept in laboratory cultures for many generations, and which may have had their ethological isolation weakened.

The changes in the chromosomal pools of the $D$. pseudoobscura populations, before and after the other species were eliminated, are shown in table 1. The composition of the sample of the wild population at Mather, California, at the time of collecting is shown in the line " 0 Days". It can be seen that,

TABLE 1

Frequencies, in percentages, of chromosomes with different gene arrangements in experimental populations of Drosophila pseudoobscura

\begin{tabular}{|c|c|c|c|c|c|c|c|}
\hline Days & $\mathrm{ST}$ & AR & TL & $\mathrm{CH}$ & PP & OL & $n$ \\
\hline Initial 0 & $37 \cdot 8$ & $44 \cdot 6$ & $11 \cdot 9$ & $3 \cdot 2$ & $2 \cdot 2$ & 0.3 & 312 \\
\hline \multicolumn{8}{|c|}{ Population I } \\
\hline $\begin{array}{r}35 \\
90 \\
150 \\
218 \\
420\end{array}$ & $\begin{array}{l}41 \cdot 3 \\
50 \cdot 0 \\
59 \cdot 3 \\
61 \cdot 7 \\
72 \cdot 7\end{array}$ & $\begin{array}{l}42 \cdot 0 \\
38 \cdot 7 \\
33 \cdot 3 \\
34 \cdot 3 \\
26 \cdot 0\end{array}$ & $\begin{array}{r}11.0 \\
6.3 \\
4.7 \\
3.3 \\
0.7\end{array}$ & $\begin{array}{l}3 \cdot 7 \\
1 \cdot 7 \\
1.3 \\
0 \cdot 3 \\
0 \cdot 3\end{array}$ & $\begin{array}{l}2 \cdot 0 \\
3.3 \\
1.3 \\
0.3 \\
0.3\end{array}$ & $\begin{array}{c}- \\
- \\
-\end{array}$ & $\begin{array}{l}300 \\
300 \\
300 \\
300 \\
300\end{array}$ \\
\hline \multicolumn{8}{|c|}{ Population II } \\
\hline $\begin{array}{r}35 \\
96 \\
155 \\
225 \\
410\end{array}$ & $\begin{array}{l}37 \cdot 7 \\
53 \cdot 3 \\
61 \cdot 3 \\
70 \cdot 3 \\
79 \cdot 0\end{array}$ & $\begin{array}{l}38 \cdot 0 \\
31 \cdot 3 \\
29 \cdot 3 \\
23 \cdot 0 \\
18 \cdot 0\end{array}$ & $\begin{array}{r}17 \cdot 7 \\
9 \cdot 0 \\
6 \cdot 0 \\
3 \cdot 3 \\
2 \cdot 0\end{array}$ & $\begin{array}{l}4 \cdot 3 \\
2 \cdot 3 \\
0 \cdot 3 \\
2 \cdot 3 \\
-\end{array}$ & $\begin{array}{l}1 \cdot 7 \\
4 \cdot 0 \\
3 \cdot 0 \\
1 \cdot 0 \\
1 \cdot 0\end{array}$ & $\begin{array}{l}0.7 \\
- \\
- \\
-\end{array}$ & $\begin{array}{l}300 \\
300 \\
300 \\
300 \\
300\end{array}$ \\
\hline
\end{tabular}

in both replicate experimental populations, the frequencies of ST chromosomes rose, while those of all other kinds declined. This is in agreement with the results of Anderson, Dobzhansky and Kastritsis (1967) for experimental populations descended from wild progenitors collected in the Pacific Coast states. However, in the present experiment, ST chromosomes were still far from fixation 410-420 days from the start; AR, TL, CH, and PP chromosomes continued to occur, although with reduced frequencies.

\section{Statistical analysis}

Statistical techniques for estimation of the Darwinian fitnesses of karyotypes from data like those in table 1 have been developed by Wright and Dobzhansky (1946), by Levene (Dobzhansky and Levene, 1951; Levene, Pavlovsky and Dobzhansky, 1954), DuMouchel and Anderson (1968), and others. We have used the technique of the authors last named. Several of the chromosomes ( $\mathrm{CH}, \mathrm{PP}$, and OL in particular) are rare in the populations, and their low frequencies pose special difficulties in the fitness estimation. We should like to combine some of the rarer types and treat them as a single class. When are we justified in doing so? 
Clearly, if two alleles (or chromosomes) act alike under selection, then we may treat them as a single class. Let us say that two alleles (or chromosomes) are selectively equivalent if the fitness values of all combinations of the two, with themselves and all other alleles in the population, are identical. We shall consider only the situation where the fitnesses are constant; the fitness of the genotype carrying alleles $i$ and $j$ is $W_{i j}$ and $W_{i j} \equiv W_{j i}$.

Alleles $m$ and $n$ are selectively equivalent if $W_{m i}=W_{n i}$ for $i=1, \ldots, k$, where $k$ is the number of alleles segregating at the locus under study. Moreover, from the basic recurrence relation for gene frequencies in successive generations,

$$
\frac{p_{m}(t)}{p_{n}(t)}=\frac{p_{m}(t-1) \sum_{j} p_{j}(t-1) W_{m j} / \bar{W}}{p_{n}(t-1) \sum_{j} p_{j}(t-1) W_{n j} / \bar{W}}=\frac{p_{m}(t-1)}{p_{n}(t-1)}
$$

where $p_{j}(t)$ is the frequency of the $j$ th allele at generation $t$ and

$$
\bar{W}=\sum_{i} \sum_{j} p_{i}(t-1) p_{j}(t-1) W_{i j}
$$

Hence, $\quad \frac{p_{m}(t)}{p_{n}(t)}=\frac{p_{m}(t-1)}{p_{n}(t-1)}=\frac{p_{m}(t-2)}{p_{n}(t-2)}=\ldots=\frac{p_{m}(0)}{p_{n}(0)}=C$,

where $C$ is a constant. It is extremely unlikely that anything other than selective equivalence could produce a constant ratio of allelic frequencies (see DuMouchel and Anderson, 1968). Thus, if the frequencies of two alleles remain in constant ratio, we may assume that the alleles are selectively equivalent in the particular experiment being considered.

Selective equivalence is easily tested by computing a homogeneity chisquare between the numbers of alleles of the two kinds observed at different generations. The frequencies of selectively equivalent alleles will be homogenous, of course, since the number of one allele in any generation is a simple multiple of the number of the other. If the probability associated with the chi-square test for homogeneity between the frequencies of two alleles exceeds some specified level, say 0.05 , then the data are consistent with the hypothesis of selective equivalence of the two alleles. The two alleles may be considered to act alike under selection, and the frequencies of the two may be combined for purposes of estimating the selection on the genotypes in the population.

The frequencies of $\mathrm{CH}, \mathrm{PP}$ and $\mathrm{OL}$ in our samples are too low for valid tests of homogeneity, and these chromosomes have accordingly been grouped into one class, which we designate $\mathrm{CH}^{*}$. The frequency of this group was then tested against those of the other chromosomes in homogeneity tests. The frequencies of TL and $\mathrm{CH}^{*}$ were very nearly in a constant ratio in each population, the probability levels associated with the tests for homogeneity being 0.1 or higher. TL and $\mathrm{CH}^{*}$ are selectively equivalent, and the frequencies of the two chromosomes were consequently added together to give the single class we call TL*. Tests for selective equivalence between $\mathrm{ST}, \mathrm{AR}$ and $\mathrm{TL} *$ indicated that no further combinations were justified. The fitnesses of the karyotypes were then estimated, assuming that the selection was constant, according to the maximum likelihood procedure of DuMouchel and Anderson (1968). Since the initial frequencies of the chromosomes were not known exactly, they were estimated jointly with the fitnesses. The chromosomal frequencies expected with these estimates of the 
fitnesses and initial frequencies were then computed, and chi-square tests for goodness of fit between observed and expected frequencies were calculated. The stable equilibrium frequencies of the chromosomes were also calculated. These results are presented in table 2 .

TABLE 2

Foint maximum likelihood estimates of karyotypic adaptive values and initial chromosomal frequencies, chi-squares for goodness of fit between observed and expected chromosomal frequencies, and predicted equilibrium frequencies

\begin{tabular}{|c|c|c|c|c|c|}
\hline \multicolumn{3}{|c|}{ Adaptive values } & \multicolumn{3}{|c|}{ Tests for goodness of fit, 5 d.f. } \\
\hline & Pop. I & Pop. II & & Pop. I & Pop. II \\
\hline $\mathrm{ST} / \mathrm{ST}$ & 0.94 & 0.93 & $\chi^{2} \mathrm{ST}$ & $0 \cdot 48$ & $2 \cdot 23$ \\
\hline ST/AR & $1 \cdot 00$ & $1 \cdot 00$ & $\chi^{2} \mathrm{AR}$ & 0.75 & 0.59 \\
\hline $\mathrm{ST} / \mathrm{TL} *$ & 0.83 & 0.78 & $\chi^{2} \mathrm{TL} *$ & 0.90 & $7 \cdot 89$ \\
\hline $\mathrm{AR} / \mathrm{AR}$ & $0 \cdot 73$ & $0 \cdot 50$ & Total $\chi^{2}$ & $2 \cdot 13$ & $10 \cdot 71$ \\
\hline $\mathrm{AR} / \mathrm{TL}^{*}$ & $0 \cdot 60$ & 0.66 & Probability & $0 \cdot 8-0.9$ & $0 \cdot 05-0 \cdot 1$ \\
\hline $\mathrm{TL} * / \mathrm{TL} *$ & $0 \cdot 76$ & 0.79 & & & \\
\hline \multicolumn{3}{|c|}{ Initial frequencies } & \multicolumn{3}{|c|}{ Predicted equilibrium frequencies } \\
\hline & Pop. I & Pop. II & & Pop. I & Pop. II \\
\hline ST & 0.371 & $0 \cdot 348$ & ST & 0.832 & $0 \cdot 879$ \\
\hline $\mathrm{AR}$ & 0.442 & 0.444 & $\mathrm{AR}$ & $0 \cdot 168$ & $0 \cdot 121$ \\
\hline TL* & 0.187 & $0 \cdot 208$ & TL* & 0.000 & 0.000 \\
\hline
\end{tabular}

$$
\mathrm{TL}^{*}=\mathrm{TL}+\mathrm{CH}+\mathrm{PP}+\mathrm{OL} \text {. }
$$

The chi-square tests indicate an excellent agreement between observed and expected frequencies in population I, and a reasonably good fit in population II. The hypothesis of constant selection seems justified for these populations. The variance-covariance matrices associated with the fitness estimates for the two populations were used to test the difference between the sets of fitness for populations I and II (DuMouchel and Anderson, 1968). The difference was small and, as might be expected from the similarity between the sets of fitnesses, nowhere near statistical significance. The selection has been quite similar in the two populations. Only the ST and AR chromosomes are expected to remain in the populations when stable equilibria are reached, the equilibrium frequency of ST being somewhere between 80 and 90 per cent.

\section{Discussion}

A variety of Drosophila species, and a variety of chromosomal forms within these species, coexist at Mather, in the Sierra Nevada of California. At least four of these species (D. pseudoobscura, D. persimilis, D. miranda, and $D$. azteca) can be maintained also in the environments of laboratory population cages. So can be populations monomorphic for ST, AR, TL, CH, and PP third chromosomes in D. pseudoobscura (OL chromosomes are too rare, and have not been tested in monomorphic populations). By contrast, when the species are made to compete in the same population cage, $D$. pseudoobscura displaces the others within at most three generations. The relative frequencies of the chromosomal forms established by natural selection in experimental populations are quite different from those in nature, ST chromosomes becoming most frequent. It is clear that selection in the 
laboratory populations is markedly different from that in nature. The estimates of fitness obtained from the experimental data suggest that only ST and AR chromosomes would be retained, and the others would be eliminated, if the selection were continued to equilibrium. This expectation is based on the assumption that the fitnesses of the karyotypes are not frequencydependent. Some karyotypes may, however, be favoured when they are rare, and at least the TL chromosomes might be retained at a low frequency.

The fact that the relative fitnesses of the karyotypes in $D$. pseudoobscura are quite different in natural and in experimental environments could not have been predicted with confidence. The chromosomal polymorphisms in some species of Drosophila are flexible, and respond easily to environmental alterations by frequency changes; the polymorphisms in other species are rigid, and are maintained despite environmental alterations (Dobzhansky, 1962). While $D$. pseudoobscura is an example of a flexible polymorphism, its Old World relative, $D$. subobscura, seems to have a relatively more rigid chromosomal polymorphism (Krimbas, 1967).

\section{Summary}

1. Two replicate experimental populations were made with four Drosophila species, namely $D$. persimilis, $D$. pseudoobscura, $D$. azteca and $D$. miranda, with initial frequencies resembling those in a natural locality in California. $D$. pseudoobscura rapidly displaced the others.

2. This species is polymorphic for gene arrangements on the third chromosome. The frequencies of the gene arrangements among the founders of the experimental populations were close to those found in nature; in the experimental environments these frequencies became sharply altered. Estimates of the selection on the karyotypes of $D$. pseudoobscura were obtained. The experimental observations fit a model of constant selection well, and the pattern of selection was quite similar in the two populations.

3. The selection in the experimental populations resulted in a loss or drastic reduction in the frequency of several of the gene arrangements, and this sharp difference between selection in natural and experimental environments serves to emphasise the flexibility of the chromosomal polymorphism in $D$. pseudoobscura.

Acknowledgments.-This work has been supported by U.S. Public Health Service Grant R01-05055-01 (to W. W. A.); and by Contract AT-(30-1)-3096, U.S. Atomic Energy Commission and Grant GB-3585 from the National Science Foundation (to Th. D.).

\section{REFERENGES}

ANDERson, w. w., DOBZHANSKy, TH., AND KASTRITsis, c. D. 1967. Selection and inversion polymorphism in experimental populations of Drosophila pseudoobscura initiated with the chromosomal constitutions of natural populations. Evolution, 21, 664-671.

ANDERSON, w. w., OSHima, c., WATANABE, T., DOBZHANSKy, TH., AND PAVLOVSKy, o. 1968. Genetics of natural populations. XXXIX. A test of the possible influence of two insecticides on the chromosomal polymorphism in Drosophila pseudoobscura. Genetics, 58, 423-434.

AyalA, F. J. 1970. Competition, coexistence, and evolution. In M. K. Hecht and W. C. Steere (eds.), Essays in Evolution and Genetics, pp. 121-158. Appleton-Century-Crofts, New York.

DOBZHANSKY, TH. 1951. Experiments on sexual isolation in Drosophila. X. Reproductive isolation between Drosophila pseudoobscura and Drosophila persimilis under natural and under laboratory conditions. Proc. Nat. Acad. Sci. U.S.A., 37, 792-796. 
DObzhansky, тн. 1962. Rigid vs. flexible chromosomal polymorphisms in Drosophila. Am. Nat., 96, 321-328.

dobzhansky, тн. 1970. Genetics of the Evolutionary Process. Columbia University Press, New York. $505 \mathrm{pp}$.

DOBZHANSKY, TH., AND LEVENE, H. 1951. Development of heterosis through natural selection in experimental populations of Drosophila pseudoobscura. Am. Nat., 85, 247-264. DUMOUCHEL, W. H., AND ANDERSON, w. w. 1968. The analysis of selection in experimental populations. Genetics, 58, 435-449.

KRIMBAS, C. B. 1967. The genetics of Drosophila subobscura populations. III. Inversion polymorphism and climatic factors. Mol. Gen. Genet., 99, 133-150.

LEVENE, H., PAVLOVSKY, O., AND DOBZHANSKY, TH. 1954. Interaction of the adaptive values in polymorphic experimental populations of Drosophila pseudoobscura. Evolution, 8, 335-349.

LEVENE, H., PAVLOVSKY, O., AND DOBZHANSKY, TH. 1958. Dependence of the adaptive values of certain genotypes in Drosophila pseudoobscura on the composition of the gene pool. Evolution, 12, 18-23.

METTLER, L. E. 1957. Studies on experimental populations of Drosophila arizonensis and Drosophila mojavensis. Univ. Texas Publ., 5721, 157-181.

PAVLOVSKY, O., AND DOBZHANSKY, TH. 1966. Genetics of natural populations. XXXVIII. The coadapted system of chromosomal variants in a population of Drosophila pseudoobscura. Genetics, 53, 843-854.

VAN VALEN, L. 1963. Introgression in laboratory populations of Drosophila persimilis and Drosophila pseudoobscura. Heredity, 18, 204-214.

WATANABE, T., ANDERSON, w. W., DOBZhansky, TH., AND PAVlovsky, o. 1970. Selection in experimental populations of Drosophila pseudoobscura with different initial frequencies of chromosomal variants. Genet. Res., 15, 123-129.

WRIGHT, s., AND DOBZHANSKY, TH. 1946. Genetics of natural populations. XXI. Experimental reproduction of some of the changes caused by natural selection in certain populations of Drosophila pseudoobscura. Genetics, 31, 142-160. 